\title{
Scoliosis surgery in adulthood: what challenges for what outcome?
}

\author{
Yann Philippe Charles, Yves Ntilikina
}

Service de Chirurgie du Rachis, Hôpitaux Universitaires de Strasbourg, Fédération de Médecine Translationnelle (FMTS), Université de Strasbourg, Strasbourg, France

Contributions: (I) Conception and design: All authors; (II) Administrative support: None; (III) Provision of study materials or patients: None; (IV) Collection and assembly of data: None; (V) Data analysis and interpretation: None; (VI) Manuscript writing: All authors; (VII) Final approval of manuscript: All authors.

Correspondence to: Yann Philippe Charles, MD, PhD. Service de Chirurgie du Rachis, Hôpitaux Universitaires de Strasbourg, Fédération de Médecine Translationnelle (FMTS), Université de Strasbourg, 1 Avenue Molière, 67200 Strasbourg, France.

Email: YannPhilippe.CHARLES@chru-strasbourg.fr.

\begin{abstract}
Adolescent idiopathic scoliosis that has progressed over time, de novo scoliosis, and degenerative scoliosis represent different types of adult spinal deformity (ASD). Functional impairment and muscular fatigue are due to sagittal and coronal imbalance of the trunk. Surgical treatment can provide a significant improvement of three-dimensional (3D) thoracolumbar alignment, function, and health-related quality of life (QoL). A patient-specific benefit-risk assessment, including clinical expectations, comorbidities, and the spinal deformity itself, has to be done preoperatively since the risk for mechanical complications is relatively high. Minimal invasive techniques combine posterior percutaneous instrumentation and lateral interbody fusion cages which enables vertebral realignment and indirect foraminal stenosis decompression. This strategy seems appropriate in mild and moderate ASD with a limited number of degenerated segments in the lumbar spine and remaining curve flexibility. Severe ASD needs to be addressed by open surgery, which combines posterior instrumentation, interbody fusion, and osteotomies in stiff deformities. Longer posterior instrumentation of the thoracolumbar spine, the sacrum, and the pelvis carries a risk for mechanical complications such as non-union and proximal junctional kyphosis (PJK). Modern surgical techniques including circumferential lumbosacral fusion and double rods might lower the risk for non-union. Accurate sagittal alignment planning, setting the lumbar sagittal apex according to pelvic incidence, and segmental lordosis distribution, are mandatory for minimizing the risk of PJK.
\end{abstract}

Keywords: Adult scoliosis; spinal deformity; sagittal malalignment; spinal stenosis; surgery; spinal instrumentation; complications

Submitted Sep 22, 2019. Accepted for publication Oct 17, 2019.

doi: 10.21037/atm.2019.10.67

View this article at: http://dx.doi.org/10.21037/atm.2019.10.67

\section{Introduction}

Adolescent idiopathic scoliosis that has progressed over time, de novo scoliosis, and lumbar degenerative scoliosis are the most common types of three-dimensional (3D) deformities among adult spinal deformity (ASD). In the aging population, intervertebral disc degeneration, facet joint osteoarthritis, paravertebral muscle dystrophy with fat infiltration, postmenopausal ligament laxity, and osteopenia all represent factors that might lead to an ASD. Adult scoliosis is usually associated with the loss of lumbar lordosis and a kyphotic deformity at the thoracolumbar junction (Figure 1). This leads to anterior imbalance of the trunk, which is compensated by retroversion of the pelvis and flexion of the knees. Sagittal malalignment in adult scoliosis has an impact on health-related quality of life (QoL) since 

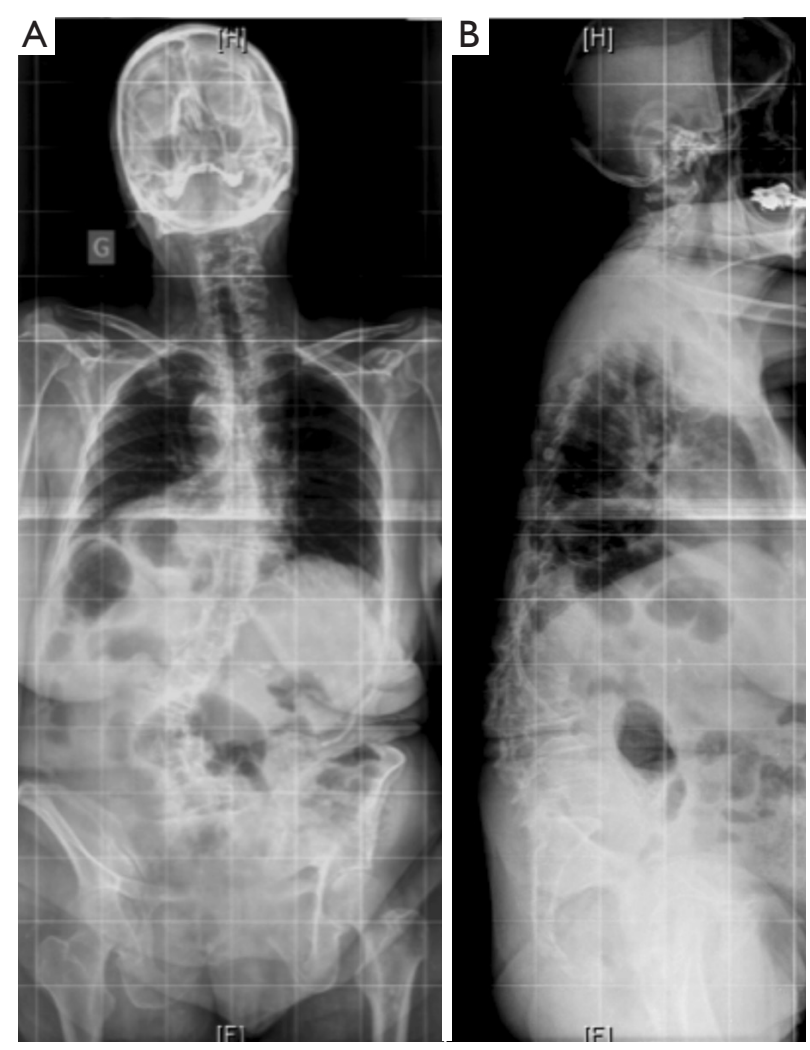

Figure 1 (A) Postero-anterior full spine radiograph showing a degenerative scoliosis and a pelvic tilt in the coronal plane, (B) the lateral full spine radiograph demonstrates a loss of lumbar lordosis and a kyphosis at the thoracolumbar junction (sagittal malalignment), which is compensated by retroversion of the pelvis and thoracic lordosis.

the energy expenditure of paravertebral muscles increases to counteract anterior imbalance, leading to back pain, fatigue and functional impairment $(1,2)$.

Degenerative changes of the lumbar spine may also lead to canal and foraminal stenosis in adults with scoliosis. More than $90 \%$ of patients that present radicular pain have a foraminal or lateral recess stenosis on magnetic resonance imaging (MRI) or computed tomography (CT), and it is usually located at level with intervertebral rotation and dislocation (3). Different patterns of stenosis due to lateral subluxation have been described (4). In open subluxations, the intervertebral disc is open towards the side of lateral displacement. This pattern results in contralateral lateral recess and foraminal stenosis in the curve concavity. In closed subluxations, the intervertebral disc is impinged towards the side of lateral displacement. This type demonstrates an ipsilateral pattern of stenosis (Figure 2).

Surgical treatment might be considered in patients with significant low back and leg pain if conservative treatment fails. Severe or progressive deformity with trunk imbalance represents and indication for instrumented deformity correction and spinal fusion $(5,6)$. In severe scoliosis with sagittal malalignment, Smith-Petersen or Ponte osteotomies might be indicated if the intersomatic space remains mobile, whereas an asymmetric pedicle subtraction osteotomy (PSO) might be used in severely rigid deformities (7). It has been demonstrated that surgical treatment of adult scoliosis can improve QoL in the mid- and long-term (8). Nevertheless, a broad range of surgical techniques exists, and patientspecific planning has to take several factors into account: age and clinical health status, comorbidities, and the $3 \mathrm{D}$ spinal deformity itself (9).

ASD surgery has increased in the aging population within the last decade despite relatively high complication rates. The overall incidence of postoperative complications is reported to be between $13 \%$ at 1 -year follow-up and $30 \%$ at 5 -year follow-up $(8,10)$. Mechanical complications represent the main reason for reoperation, and their incidence among all complications is reported to be between $30 \%$ and $40 \%(11,12)$. The most common failures related to thoracolumbar instrumentation, including the lumbosacral junction and pelvis, are non-union and rod breakage as well as proximal junctional kyphosis (PJK) and pedicle screw loosening. It is therefore mandatory to complete accurate benefit-risk assessment and preoperative planning to avoid instrumentation failure, which negatively affects clinical outcomes in the event of multiple revision surgeries (13).

This review will describe the principles of surgical management of adult scoliosis using either minimal invasive surgery (MIS) or classic posterior deformity correction and instrumentation combined with anterior interbody fusion. Specific aspects of non-union and PJK will be emphasized and avoidance strategies of mechanical complications will be outlined.

\section{MIS}

The past decade has seen major advances for treating ASD using MIS techniques. Minimal invasive techniques usually combine posterior percutaneous instrumentation and anterior retroperitoneal approaches using interbody fusion cages. This combined MIS approach has the advantage of sparing paravertebral muscle dissection, minimal blood 

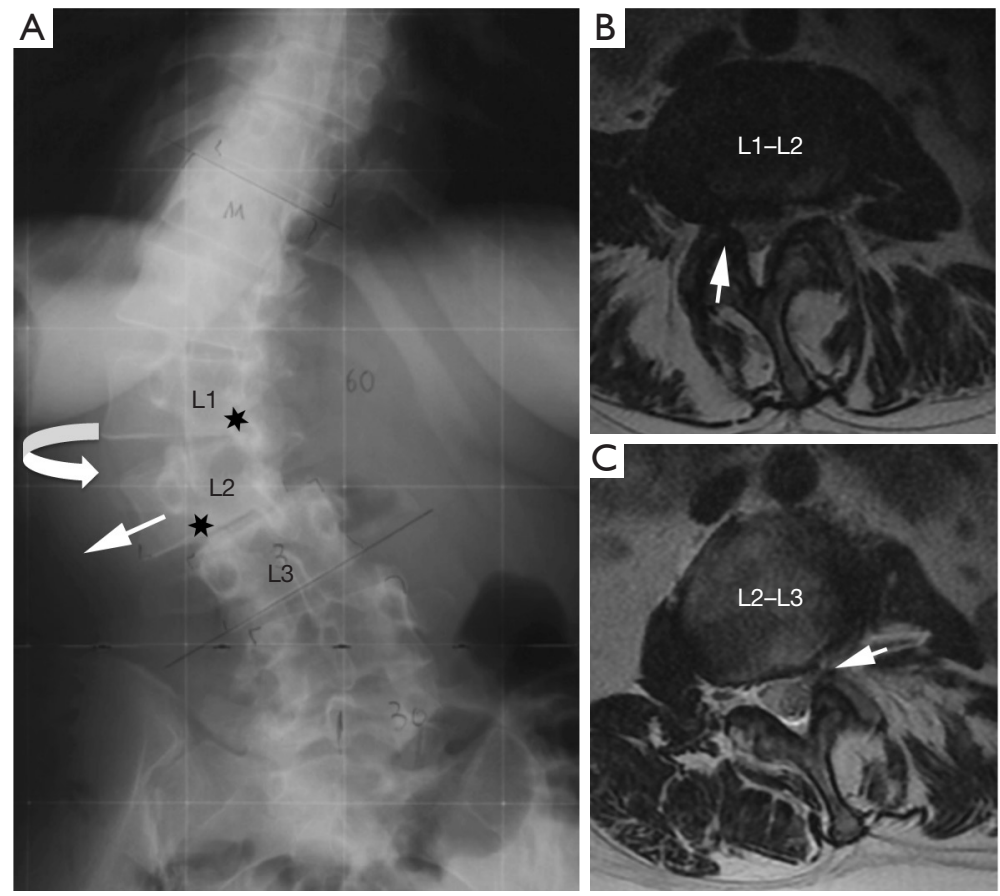

Figure 2 (A) Posterior-anterior lumbar radiograph showing vertebral rotation at L1-L2 with disc opening towards the curve convexity and lateral subluxation at L2-L3; stars indicate levels of stenosis; (B) axial MRI showing lateral recess stenosis at L1-L2, open subluxation and (C) foraminal stenosis at L2-L3, and closed subluxation. MRI, magnetic resonance imaging.

loss, and shorter operative time compared to open surgery. Experienced surgeons are treating older and more morbid patients with similar outcomes in mild and moderate spinal deformities (14). Sagittal deformity correction mainly relies on powerful interbody reduction methods that imply a resection of the degenerated intervertebral disc and anterior longitudinal ligament. Coronal plane deformities with asymmetric interbody spaces can also be corrected by lateral cage insertion, thus orientating vertebral endplates in parallel. At L5-S1, anterior interbody lumbar fusion (ALIF) cages with a large footprint are used, which creates lordosis at the lumbosacral junction. At the levels from T11-T12 to L4-L5, oblique lateral interbody fusion (OLIF) or extreme lateral interbody fusion (XLIF) cages are used. With the OLIF technique, the psoas muscle is reclined, including the femoral nerve, whereas the XLIF technique requires electromyography monitoring to detect the nerve as the approach is made through the psoas muscle $(15,16)$.

Segmental intervertebral height increases and lordosis restoration produces an indirect decompression of spinal stenosis at the level of the lateral recess by tethering the bulging disc and flavum ligament. Furthermore, the cage implantation produces a cranial-caudal distraction of the impinged facet joints, which increases the foraminal height. This indirect anterior decompression technique enables to address open and closed subluxations without the need for an open approach to the spinal canal (17). It has been demonstrated that low back pain and leg pain improve and that the segmental lumbar deformity can be efficiently corrected with MIS techniques. $(14,18)$. This strategy seems appropriate in mild and moderate ASD which present degenerative changes of the lumbar spine and a remaining curve flexibility (19). De novo scoliosis with a single or bi-level disc degeneration represents a good indication for early MIS treatment, thus preventing further curve progression (Figures 3,4). Severe sagittal and coronal imbalance represents a limitation for MIS. Stiff deformities that cannot be reduced on bending radiographs or that present interbody fusions on CT should be addressed by open posterior surgery including a facet joint release, osteotomies, and interbody fusion.

\section{Posterior instrumentation}

Open posterior instrumentation is based on segmental pedicle screw-rod fixation. Different correction strategies 

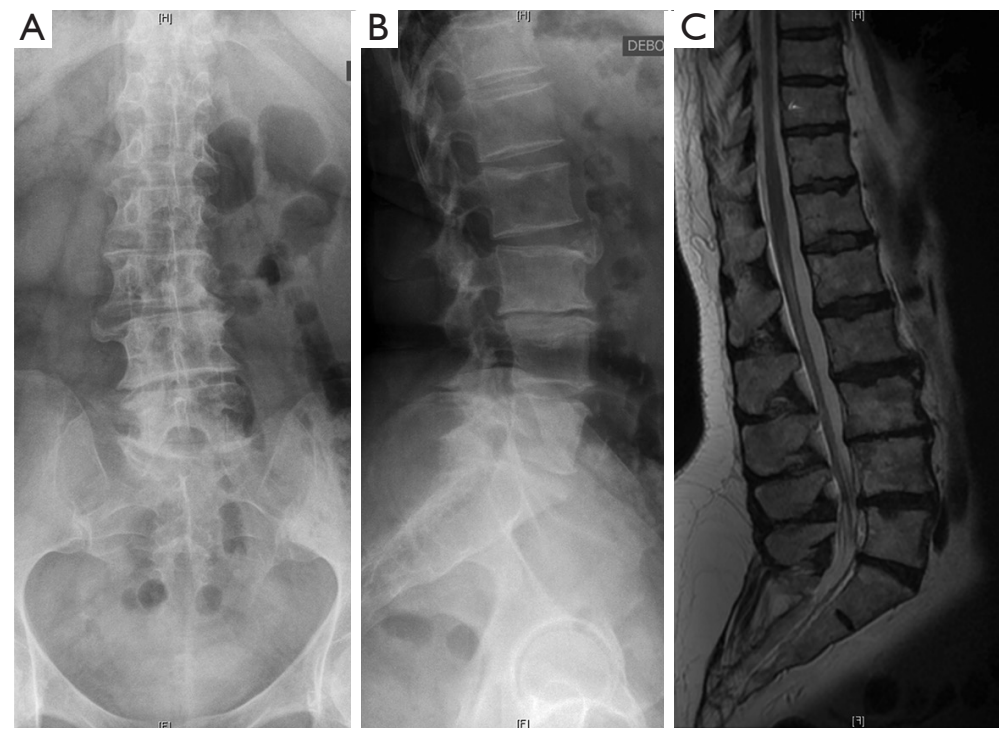

Figure 3 (A) Anterior-posterior and (B) sagittal lumbar radiographs, (C) MRI with sagittal reconstruction showing intervertebral disc degeneration and a beginning de novo scoliosis at L3-L4. MRI, magnetic resonance imaging.
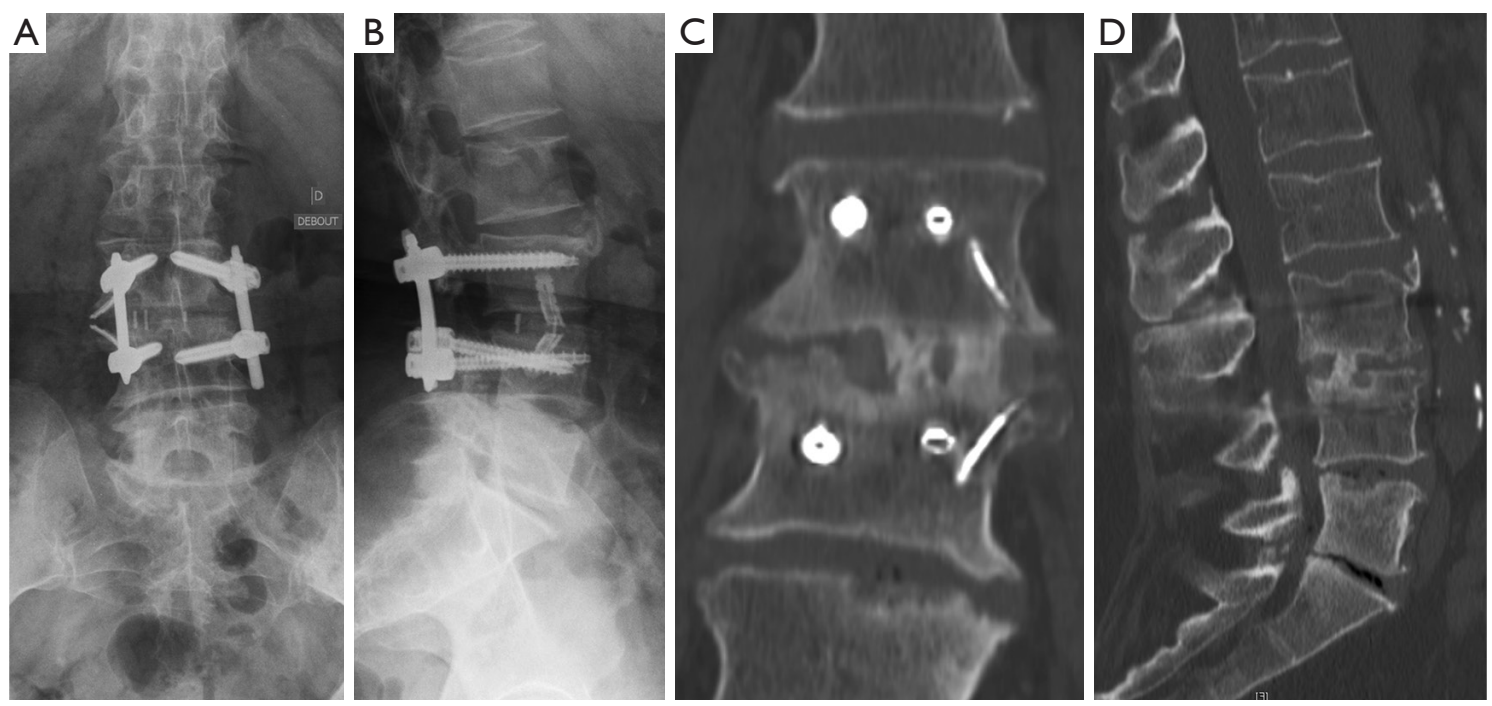

Figure 4 Postoperative (A) posterior-anterior and (B) lateral radiographs showing lumbar interbody fusion combined with posterior percutaneous instrumentation at L3-L4; 1-year follow-up CT showing interbody fusion through the OLIF cage on (C) coronal and (D) sagittal reconstructions (same patient as in Figure 3). CT, computed tomography; OLIF, oblique lateral interbody fusion.

exist for the reduction of severe scoliosis. Modern approaches usually combine reduction maneuvers such as direct vertebral derotation, rod translation, and approximation using persuader systems (20). In situ bending represents an additional technique that uses a rod that is first connected to the pedicle screws following the shape of spinal deformity (21). The rod is then bent by sequential maneuvers in the coronal and sagittal planes. Monoaxial screws are placed on most rotated vertebrae on the convex side of the lumbar curve. This technique will allow rotating of the lumbar apex using levers during sequential bending maneuvers of the rod and lead to a $3 \mathrm{D}$ correction of the deformity (Figure 5).

Furthermore, a preoperative analysis of residual 

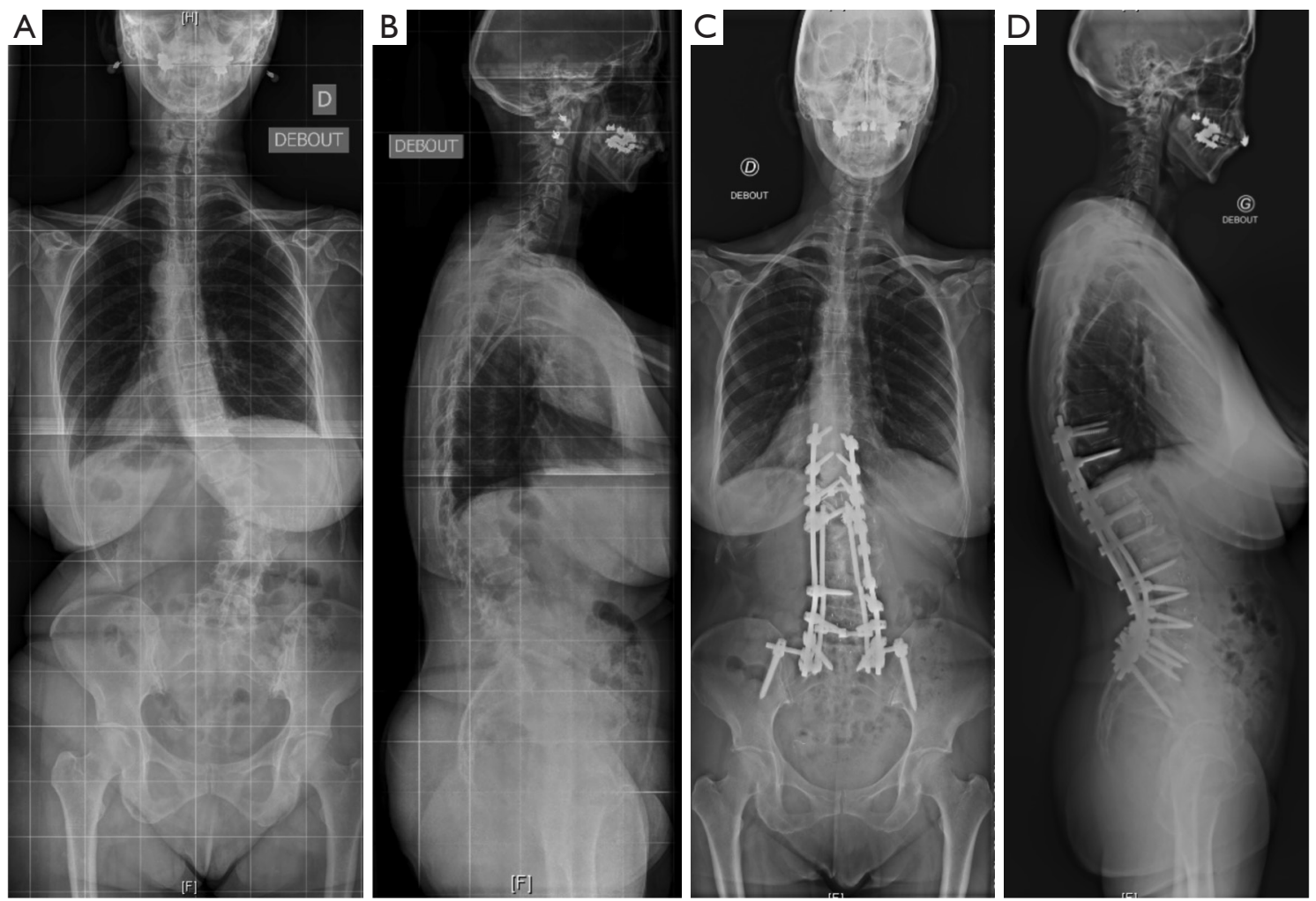

Figure 5 Preoperative (A) posterior-anterior and (B) lateral full spine radiographs of a patient with lumbar degenerative scoliosis, postoperative (C) coronal and (D) sagittal correction by posterior instrumentation, and fusion from T10 to the pelvis.

curve flexibility on side bending radiographs and CT is mandatory. A posterior facet joint release can be performed prior to correction in order to increase the flexibility of the spinal deformity. This release may be completed by Ponte osteotomies for segmental kyphosis correction. This technique may improve segmental sagittal alignment in segments that are non-fused by interbody osteophytes. Severe rigid deformities with interbody fusion areas usually require a posterior three-column osteotomy such as an asymmetric PSO $(7,19,20)$.

Posterior instrumentation in ASD usually includes the sacrum since L5-S1 disc degeneration and facet joint osteoarthritis are present in most patients. However, the optimal distal fixation point has been a matter of debate because screw loosening might occur on long-term in long posterior instrumentation $(22,23)$. S1 pedicle screws should follow a convergent axis into the promontorium with a bicortical fixation. This technique enhances the screw purchase. An additional fixation of the instrumentation at the pelvis might decrease the risk for S1 screw loosening (24). S2-alar screws represent an alternative to ilium screws thus avoiding far lateral paravertebral and gluteus muscle dissection in the sacro-pelvic area. The S2-alar screw entry point is caudal to the posterior $\mathrm{S} 1$ foramen, and the screw axis crosses the sacroiliac joint (25). Although the rigid sacro-pelvic fixation has decreased the risk for distal screw loosening, cyclic loading during daily activities might lead to fatigue of the posterior instrumentation, which can result in mechanical long-term complications such as non-union.

\section{Non-union and rod failure}

Non-union should be suspected if the patient is in pain and if radiographs show a rod fracture (Figure 6). CT combined with $99 \mathrm{mTC}$-HMDP single positron emission tomography (SPECT-CT) should confirm the diagnosis (26). Posterior fusion without anterior column support might not be sufficient when instrumenting the thoracolumbar spine including the sacrum and pelvis. The lumbosacral junction may be fused by the anterior approach (ALIF), which has the advantage of a large cage surface and resistance under axial compression to avoid non-union and 

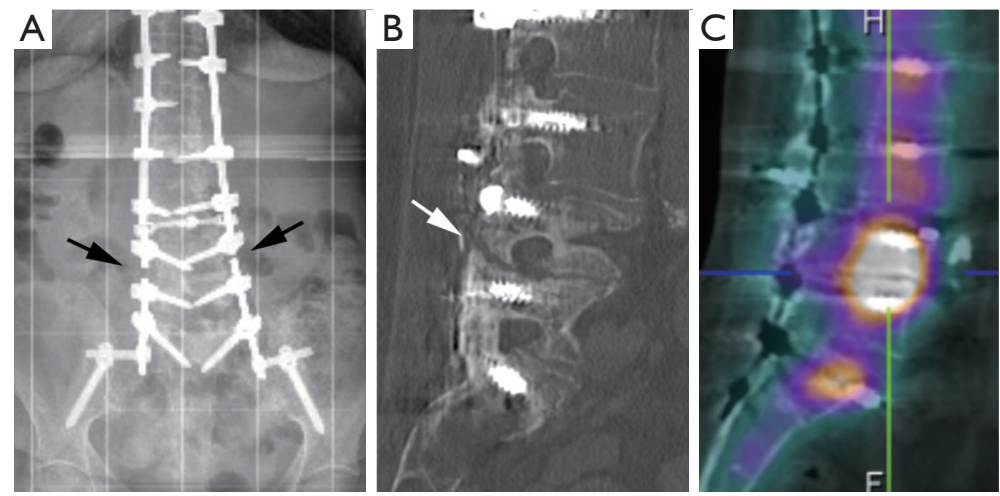

Figure 6 (A) Rod fractures on radiographs, (B) osteolysis in the fusion mass on CT and (C) hyperfixation on SPECT-CT indicate nonunion. CT, computed tomography; SPECT, single positron emission tomography.
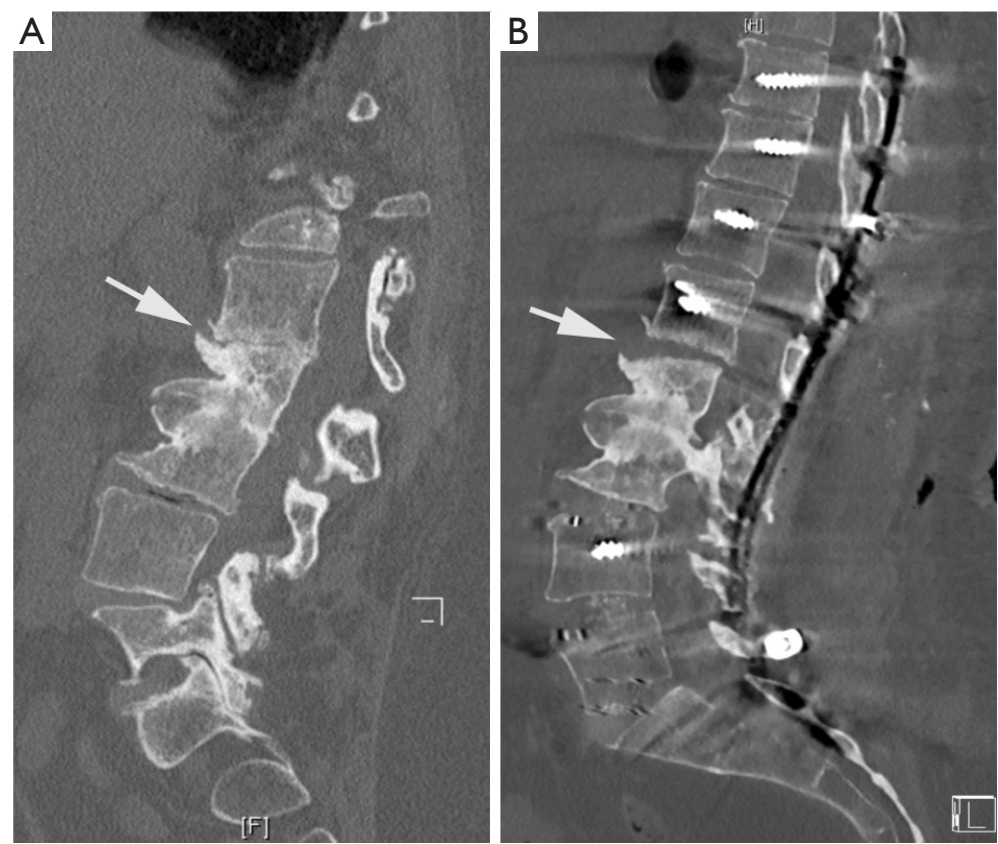

Figure 7 (A) Preoperative sagittal CT and (B) postoperative CT showing opening of the remaining L1-L2 disc space after posterior Ponte osteotomy and instrumentation. This gap needs to be filled by an anterior OLIF cage to avoid non-union. Interbody fusion has been performed using TLIF cages at L3-L4, L4-L5, and L5-S1. CT, computed tomography; OLIF, oblique lateral interbody fusion; TLIF, transforaminal interbody fusion.

subsequent revision surgery (27). The alternative would be a transforaminal interbody fusion (TLIF), which can be performed during the posterior deformity correction. Recently, dual rod techniques have been described (28-31). Their aim is to decrease strain at the level of primary rods by reinforcing the instrumentation by two additional rods when the lumbosacral junction and the pelvis are included (Figure 5).
Furthermore, the segmental kyphosis correction by posterior Ponte osteotomies will induce an anterior opening of mobile discs. In some cases, anterior osteophytes can break, and narrow disc spaces open up when increasing lordosis by powerful in situ bending maneuvers (Figure 7). It is mandatory to stabilize these segments by anterior grafting in order to prevent the loss of correction and non-union. An OLIF cage might be recommended in a second stage surgery. 


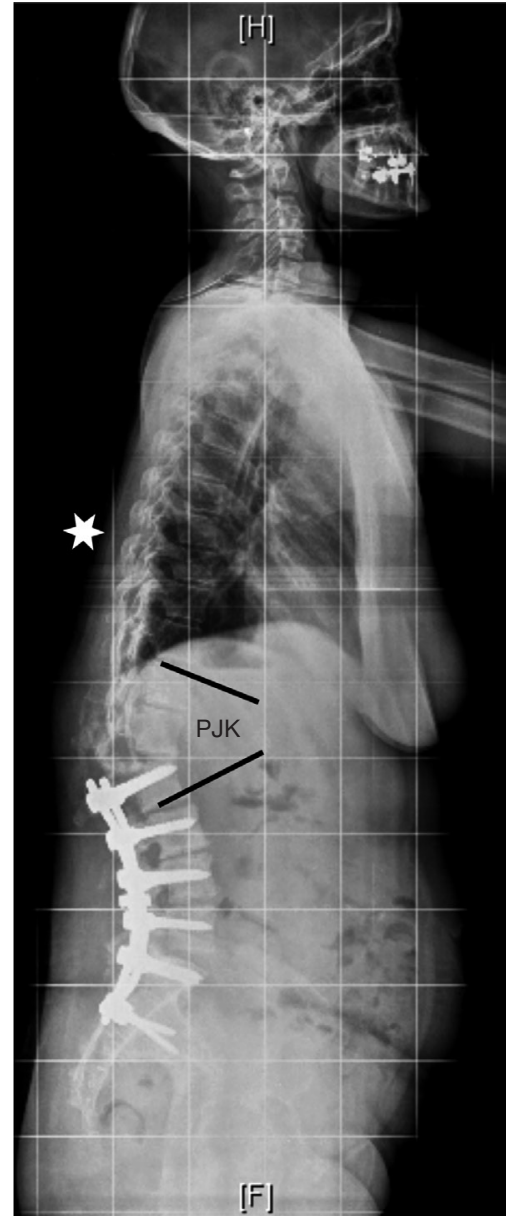

Figure 8 Sagittal full spine radiograph showing PJK due to overcorrection in a patient with low pelvic incidence. PJK, proximal junctional kyphosis.

In painful non-union revision, surgery that includes posterior and anterior fusion is usually indicated. If a rod fracture is present at one level, there is a risk for multiple level non-unions. All spinal levels should be carefully examined on imaging and tested intra-operatively in order to avoid multiple revision surgeries. In the event of a single revision surgery, the long-term clinical results can improve and the outcome might be satisfactory for the patient (8). However, the functional clinical scores decline if several revision surgeries are performed (13).

\section{PJK}

PJK represents another common complication after ASD surgery (Figure 8). It is defined by an increase of more than $10^{\circ}$ of kyphosis between the caudal endplate of the proximally instrumented vertebra and the cranial endplate of the vertebra two levels above (32). Its cause is probably multifactorial. The thoracic apex and junctional vertebrae (T12-L1) should be avoided as proximal endpoints of instrumentation to avoid PJK. Furthermore, osteoporosis and osteopenia might represent risk factors for proximal screw loosening and subsequent PJK. However, a direct relationship between decreased bone mineral density and PJK has not been proven $(33,34)$. Prophylactic cement augmentation of cranial pedicle screws and a vertebroplasty of the adjacent non-instrumented vertebral body might represent a method to avoid toggle migration of the screws and vertebral compression fractures (Figure 9).

Furthermore, a patient and age specific sagittal alignment needs to be considered when planning a posterior deformity correction (35). The spino-pelvic organization depending on pelvic incidence and sacral slope will influence the lumbar lordosis distribution between the upper and lower lumbar spine. Roussouly has classified the sagittal alignment into four types (36). Type 1 has a small pelvic incidence and sacral slope; the lumbar lordosis has a short caudal arch with an apex at L5. Type 2 has a small pelvic incidence and sacral slope too, but the lordosis apex is higher at the basis of L4. Type 3 has an intermediate pelvic incidence and sacral slope; lumbar lordosis is more prominent with an apex at the center of L4. A subtype characterized by an anteverted pelvis exists in this category. Type 4 has a high pelvic incidence and sacral slope with a large lordosis and an apex at the basis of L3. If the sagittal apex of lumbar lordosis is set too proximally according to the spinopelvic organization, it is very likely that $\mathrm{PJK}$ occurs $(37,38)$. The lumbar apex should not be higher than L4 if the pelvic incidence is $<55^{\circ}$. Only patients with a pelvic incidence $>55^{\circ}$ can tolerate an apex at the L3-L4 disc or L3. Similarly, the global alignment proportion (GAP) score is based on lordosis distribution, relative lumbar lordosis and global tilt according to pelvic incidence, and relative pelvic version and age (39). In patients where sagittal alignment restoration fails, the target of proportionate lordosis distribution, the risk of PJK, and mechanical instrumentation failure increases. The Roussouly classification and the GAP score are valuable tools that should be considered preoperatively in order to determine the optimal correction strategy and sagittal profile of the instrumented spine.

\section{Conclusions}

Adult scoliosis correction provides an improvement of 

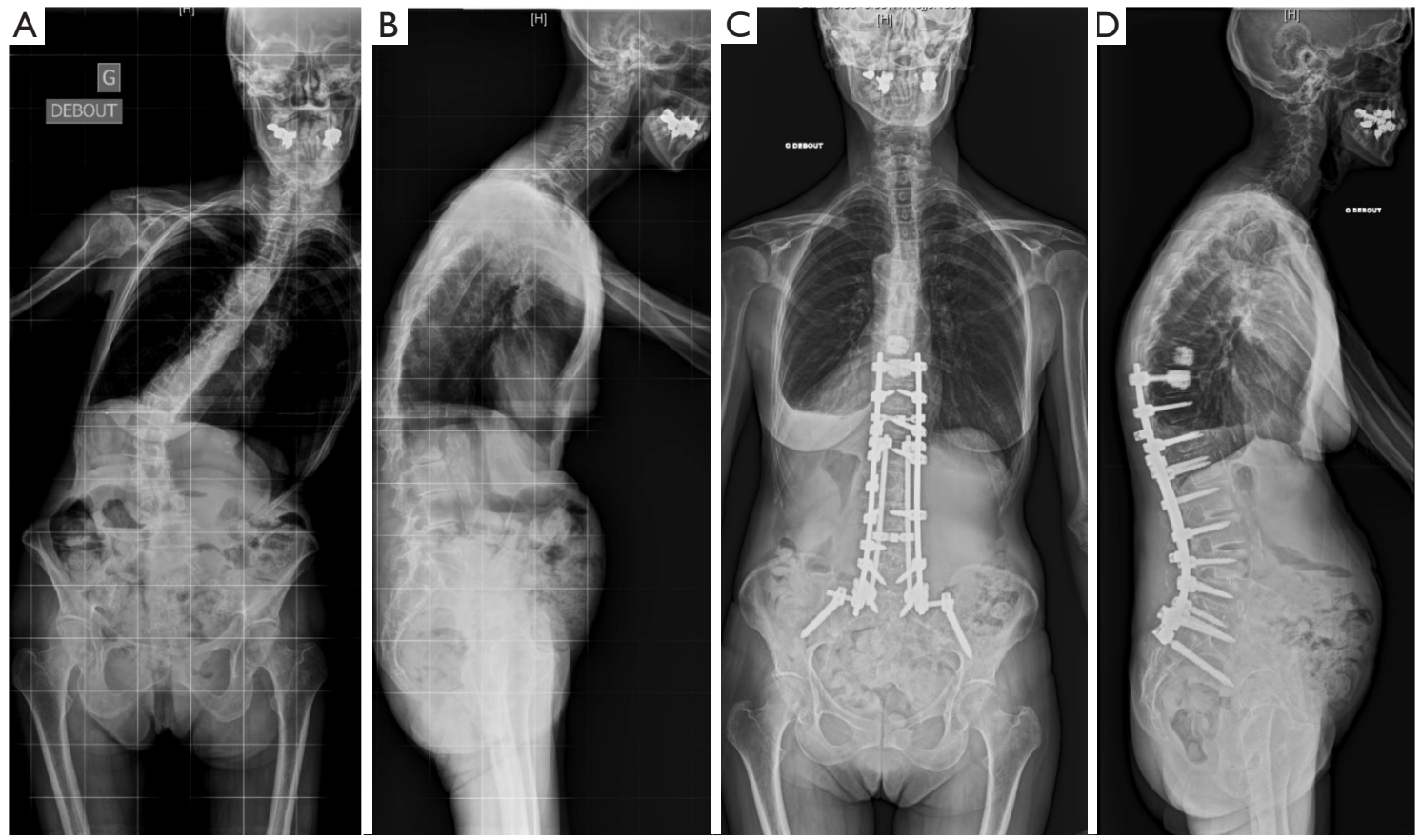

Figure 9 Preoperative (A) posterior-anterior and (B) lateral full spine radiographs of a patient with adult scoliosis and osteopenia; postoperative (C) coronal and (D) sagittal correction by posterior instrumentation and fusion from T10 to the pelvis with cement augmentation of T9 and T10 to avoid PJK. PJK, proximal junctional kyphosis.

3D thoracolumbar alignment, function, and healthrelated QoL. A patient-specific benefit-risk assessment has to be done preoperatively since the risk for mechanical complications is relatively high. Modern technical aspects such as circumferential lumbosacral fusion and double rods might lower the risk for non-union. Accurate sagittal alignment planning is mandatory to minimize the risk for PJK.

\section{Acknowledgments}

None.

\section{Footnote}

Conflicts of Interest: The authors have no conflicts of interest to declare.

Ethical Statement: The authors are accountable for all aspects of the work in ensuring that questions related to the accuracy or integrity of any part of the work are appropriately investigated and resolved.

\section{References}

1. Smith JS, Klineberg E, Schwab F, et al. Change in classification grade by the SRS-Schwab adult spinal deformity classification predicts impact on health-related quality of life measures: prospective analysis of operative and nonoperative treatment. Spine (Phila Pa 1976) 2013;38:1663-71.

2. Schwab FJ, Blondel B, Bess S, et al. Radiographical spinopelvic parameters and disability in the setting of adult spinal deformity: a prospective multicenter analysis. Spine (Phila Pa 1976) 2013;38:E803-12.

3. Fu KM, Rhagavan P, Shaffrey CI, et al. Prevalence, severity, and impact of foraminal and canal stenosis among adults with degenerative scoliosis. Neurosurgery 2011;69:1181-7.

4. Gardner RO, Torrie PA, Bertram W, et al. A radiological evaluation of lateral vertebral subluxation associated with spinal stenosis in the lumbar spine in degenerative scoliosis. Spine Deform 2013;1:365-70.

5. Chen PG, Daubs MD, Berven S, et al. Surgery for degenerative lumbar scoliosis: the development of appropriateness criteria. Spine (Phila Pa 1976) 
2016;41:910-8.

6. Neuman BJ, Baldus C, Zebala LP, et al. Patient factors that influence decision making: randomization versus observational nonoperative versus observational operative treatment for adult symptomatic lumbar scoliosis. Spine (Phila Pa 1976) 2016;41:E349-58.

7. Enercan M, Ozturk C, Kahraman S, et al. Osteotomies/ spinal column resections in adult deformity. Eur Spine J 2013;22 Suppl 2:S254-64.

8. Kyrölä K, Kautiainen H, Pekkanen L, et al. Long-term clinical and radiographic outcomes and patient satisfaction after adult spinal deformity correction. Scand J Surg 2018. [Epub ahead of print].

9. Ames CP, Smith JS, Pellisé F, et al. Development of deployable predictive models for minimal clinically important difference achievement across the commonly used health-related quality of life instruments in adult spinal deformity surgery. Spine (Phila Pa 1976) 2019;44:1144-53.

10. Sugawara R, Takeshita K, Inomata Y, et al. The Japanese scoliosis society morbidity and mortality survey in 2014 : the complication trends of spinal deformity surgery from 2012 to 2014. Spine Surg Relat Res 2018;3:214-21.

11. Kelly MP, Lenke LG, Bridwell KH, et al. Fate of the adult revision spinal deformity patient: a single institution experience. Spine (Phila Pa 1976) 2013;38:E1196-200.

12. Zhu F, Bao H, Liu Z, et al. Unanticipated revision surgery in adult spinal deformity: an experience with 815 cases at one institution. Spine (Phila Pa 1976) 2014;39:B36-44.

13. Hu X, Lieberman IH. Revision adult spinal deformity surgery: does the number of previous operations have a negative impact on outcome? Eur Spine J 2019;28:155-60.

14. Wang MY, Tran S, Brusko GD, et al. Less invasive spinal deformity surgery: the impact of the learning curve at tertiary spine care centers. J Neurosurg Spine 2019:1-8.

15. Isaacs RE, Hyde J, Goodrich JA, et al. A prospective, nonrandomized, multicenter evaluation of extreme lateral interbody fusion for the treatment of adult degenerative scoliosis: perioperative outcomes and complications. Spine (Phila Pa 1976) 2010;35:S322-30.

16. Silvestre C, Mac-Thiong JM, Hilmi R, et al. Complications and morbidities of mini-open anterior retroperitoneal lumbar interbody fusion: oblique lumbar interbody fusion in 179 patients. Asian Spine J 2012;6:89-97.

17. Alimi M, Hofstetter CP, Tsiouris AJ, et al. Extreme lateral interbody fusion for unilateral symptomatic vertical foraminal stenosis. Eur Spine J 2015;24 Suppl 3:346-52.

18. Wang K, Zhang C, Cheng C, et al. Radiographic and clinical outcomes following combined oblique lumbar interbody fusion and lateral instrumentation for the treatment of degenerative spine deformity: a preliminary retrospective study. Biomed Res Int 2019;2019:5672162.

19. Berjano P, Lamartina C. Far lateral approaches (XLIF) in adult scoliosis. Eur Spine J 2013;22 Suppl 2:S242-53.

20. Koller H, Pfanz C, Meier O, et al. Factors influencing radiographic and clinical outcomes in adult scoliosis surgery: a study of 448 European patients. Eur Spine J 2016;25:532-48.

21. Steib JP, Dumas R, Mitton D, et al. Surgical correction of scoliosis by in situ contouring: a detorsion analysis. Spine (Phila Pa 1976) 2004;29:193-9.

22. Banno T, Hasegawa T, Yamato Y, et al. Prevalence and risk factors of iliac screw loosening after adult spinal deformity surgery. Spine (Phila Pa 1976) 2017;42:E1024-30.

23. Edwards CC 2nd, Bridwell KH, Patel A, et al. Long adult deformity fusions to L5 and the sacrum. A matched cohort analysis. Spine (Phila Pa 1976) 2004;29:1996-2005.

24. Volkheimer D, Reichel H, Wilke HJ, et al. Is pelvic fixation the only option to provide additional stability to the sacral anchorage in long lumbar instrumentation? A comparative biomechanical study of new techniques. Clin Biomech (Bristol, Avon) 2017;43:34-9.

25. Koller H, Zenner J, Hempfing A, et al. Reinforcement of lumbosacral instrumentation using S1-pedicle screws combined with S2-alar screws. Oper Orthop Traumatol 2013;25:294-314.

26. Heimburger C, Hubele F, Charles YP, et al. Évaluation de critères d'interprétation de la tomoscintigraphie d'émission monophotonique au 99mTC-HMDP pour le diagnostic des complications tardives des arthrodèses rachidiennes. Med Nucl 2015;39:105-21.

27. Hlubek RJ, Godzik J, Newcomb AGUS, et al. Iliac screws may not be necessary in long-segment constructs with L5S1 anterior lumbar interbody fusion: cadaveric study of stability and instrumentation strain. Spine J 2019;19:942-50.

28. Godzik J, Hlubek RJ, Newcomb AGUS, et al. Supplemental rods are needed to maximally reduce rod strain across the lumbosacral junction with TLIF but not ALIF in long constructs. Spine J 2019;19:1121-31.

29. Hyun SJ, Lenke LG, Kim YC, et al. Comparison of standard 2-rod constructs to multiple-rod constructs for fixation across 3-column spinal osteotomies. Spine (Phila Pa 1976) 2014;39:1899-904.

30. Merrill RK, Kim JS, Leven DM, et al. Multi-rod constructs can prevent rod breakage and pseudarthrosis at the lumbosacral junction in adult spinal deformity. Global 
Spine J 2017;7:514-20.

31. Gupta S, Eksi MS, Ames CP, et al. A novel 4-rod technique offers potential to reduce rod breakage and pseudarthrosis in pedicle subtraction osteotomies for adult spinal deformity correction. Oper Neurosurg (Hagerstown) 2018;14:449-56.

32. Glattes RC, Bridwell KH, Lenke LG, et al. Proximal junctional kyphosis in adult spinal deformity following long instrumented posterior spinal fusion: incidence, outcomes, and risk factor analysis. Spine (Phila Pa 1976) 2005;30:1643-9.

33. Cammarata M, Aubin CÉ, Wang X, et al. Biomechanical risk factors for proximal junctional kyphosis: a detailed numerical analysis of surgical instrumentation variables. Spine (Phila Pa 1976) 2014;39:E500-7.

34. Yagi M, Akilah KB, Boachie-Adjei O. Incidence, risk factors and classification of proximal junctional kyphosis: surgical outcomes review of adult idiopathic scoliosis. Spine (Phila Pa 1976) 2011;36:E60-8.

Cite this article as: Charles YP, Ntilikina Y. Scoliosis surgery in adulthood: what challenges for what outcome? Ann Transl Med 2020;8(2):34. doi: 10.21037/atm.2019.10.67
35. Lafage R, Schwab F, Glassman S, et al. Age-adjusted alignment goals have the potential to reduce PJK. Spine (Phila Pa 1976) 2017;42:1275-82.

36. Roussouly P, Gollogly S, Berthonnaud E, et al. Classification of the normal variation in the sagittal alignment of the human lumbar spine and pelvis in the standing position. Spine (Phila Pa 1976) 2005;30:346-53.

37. Sebaaly A, Riouallon G, Obeid I, et al. Proximal junctional kyphosis in adult scoliosis: comparison of four radiological predictor models. Eur Spine J 2018;27:613-21.

38. Pizones J, Martin MB, Perez-Grueso FJS, et al. Impact of adult scoliosis on Roussouly sagittal shape classification. Spine (Phila Pa 1976) 2019;44:270-9.

39. Yilgor C, Sogunmez N, Boissiere L, et al. Global alignment and proportion (GAP) score: development and validation of a new method of analyzing spinopelvic alignment to predict mechanical complications after adult spinal deformity surgery. J Bone Joint Surg Am 2017;99:1661-72. 